\title{
LA CONDICIÓN BIFOLIA: LOS IMPRESOS DE DOS HOJAS YLA TRANSMISIÓN DEL PLIEGO SUELTO POÉTICO*
}

\author{
Mario Garvin \\ Universität Konstanz (Alemania) \\ mario.garvin@uni-konstanz.de
}

En un temprano trabajo sobre los pliegos sueltos poéticos quinientistas, pionero en muchos aspectos, Edward M. Wilson y Frederik J. Norton caracterizaron el pliego poético en base a «its quarto format, the restriction of its text to a single folded sheet, its title embellished by an irrelevant reused woodcut with the text beginning immediately below in two columns, its gothic types, and its lack of any indication of place, printer or date» ${ }^{1}$. Las inmensas pérdidas sufridas en este campo obligan ciertamente a la precaución, pero lo cierto es que en el momento de escribir estas líneas, 2019, medio siglo después de la afirmación de Norton y Wilson, esos siguen siendo los rasgos dominantes entre los pliegos sueltos poéticos quinientistas conocidos, cuyo corpus actual alcanza unas 1200 referencias, registradas en el Nuevo Diccionario $^{2}$ y en el posterior Suplemento ${ }^{3}$.

${ }^{*}$ Este trabajo se enmarca dentro del proyecto Cancionero, romancero y fuentes impresas, del Ministerio de Economía, Industria y Competitividad (FFI2017-86313-P), financiado por la Agencia Estatal de Investigación (AEI) y el Fondo Europeo de Desarrollo Regional (FEDER/UE), cuyo investigador principal es Josep Lluís Martos.

${ }^{1}$ Edward M. Wilson y Frederik J. Norton, Two Spanish Verse Chap-Books, Romance de Amadis (c. 1515-1519), Juizio hallado y trobado (c. 1510), Cambridge, Cambridge University Press, 1969, p. 5.

${ }^{2}$ Antonio Rodríguez-Moñino, Nuevo Diccionario Bibliográfico de pliegos sueltos poéticos (Siglo $X V I$ ), ed. actualizada y corregida por Arthur L.F. Askins y Víctor Infantes, Castalia, Nueva Biblioteca de Erudición y Crítica, 1997.

${ }^{3}$ Arthur L.F. Askins y Víctor Infantes, Suplemento al Nuevo Diccionario bibliográfico de pliegos sueltos poéticos (Siglo XVI) de Antonio Rodríguez-Moñino, ed. de Laura Puerto Moro, Madrid, Editorial Academia del Hispanismo, 2014. 
Resulta difícil esbozar las vías por las que estos elementos particulares encuentran su acomodo en el sistema que constituye el pliego suelto ${ }^{4}$. No sabemos aún a ciencia cierta, por ejemplo, la relación que se establece entre los grabados que aparecen en esos pliegos (afirmar que los decoran constituye ya en sí una valoración que no estoy seguro de compartir) y su contenido; muchos de estos grabados, además, se emplean para diversos -y diferentes- pliegos, en un juego que, en el caso de las figuritas que aparecen en no pocos de ellos ${ }^{5}$, fuerza notablemente, a base de su repetición en distintos contextos, la polisemia del impreso. Tampoco está demasiado claro a qué obedece exactamente la estandarización del formato en cuarto. En parte, la generalización de este formato estaría relacionada con los modos de lectura de estos pliegos, según parece sugerirlo el uso experimental del formato folio en algunos de ellos y, especialmente, su progresivo abandono en ediciones posteriores $^{6}$. Pero probablemente, la vía por la que mejor se aclaran formato y extensión es la relación directa que existe entre el pliego suelto poético y el pliego de papel que le sirve de base, o mejor dicho, su equivalencia, pues un pliego poético no es sino el resultado de doblar una hoja de papel en su tamaño natural las veces necesarias, dependiendo del formato elegido, para dar lugar a un pliego 7 . Y es que como dejó escrito Víctor Infantes: «En la hoja básica de impresión, el llamado "papel de marca", el pliego por antonomasia, de unos 440 x $320 \mathrm{~mm}$ aproximadamente [...] caben numerosas posibilidades, aunque siempre constreñidas por la extensión original del papel, que a lo largo de todos estos años se ampliaron en diferentes opciones y disposiciones tipográficas y editoriales» ${ }^{8}$.

\footnotetext{
${ }^{4}$ Me refiero a sistema en el sentido en que lo emplean Johanna Drucker y Jerome McGann: «I want to rework the conventional approach to the idea of the "page" as an a priori "space" for graphical construction. In its place, I want to propose an understanding of all graphical elements as dynamic entities in what Jerome McGann and I refer to as a "quantum field"-or more recently, "system"», Johanna Drucker, «Graphical readings and the visual aesthetics of textuality», en Text, 16 (2006), pp. 267-276, p. 269.

${ }^{5}$ Sobre el uso de estas figuras, véase el trabajo de Mercedes Fernández Valladares «Biblioiconografía y literatura popular impresa: la ilustración de los pliegos sueltos burgaleses (o de babuines y estampas celestinescas)», en eHumanista. Journal of Iberian Studies, 21 (2012), pp. 87-131.

${ }^{6}$ Norton y Wilson ya dejaron escrito en el mismo lugar que «at Seville the folio form was used, perhaps experimentally» y que «these folio pieces are also exceptional in being printed in four ant three columns respectivelly», p. 6.

${ }^{7}$ El proceso de producción es hoy sobradamente conocido. Remito solamente a los artículos reunidos en el volumen Imprenta y Crítica Textual en el Siglo de Oro, ed. de Francisco Rico, Valladolid, Universidad de Valladolid, Centro para la Edición de los Clásicos Españoles, 2000.

${ }^{8}$ Víctor Infantes, «Historia mínima (y desde luego incompleta) de los impresos de una sola hoja. II. Los años áureos», en Edad de oro Cantabrigense. Actas del VII Congreso de la Asociación Internacional Siglo de Oro (AISO), Robinson College, Cambridge, 18-22 julio, 2005, ed. de Anthony Close, Cambridge, Iberoamericana, 2006, pp. 351-356, p. 352.
} 
Uno de los factores que más decisivamente contribuyeron al éxito de los pliegos sueltos fue precisamente esa equivalencia material con la hoja de papel. Frente al resto de formas editoriales, el pliego poético de un solo pliego -y con él todas aquellas otras de igual extensión- era la única que no requería de más operaciones que la propia impresión y el plegado. Además, como recordaba Jaime Moll, «la tirada media de un día de trabajo son mil quinientos pliegos, lo que recibe el nombre de jornada, o sea un total de tres mil golpes por cada cara del pliego, lo que representa seis mil golpes de prensa. Actualmente nos parece una cifra exagerada, pero esa era la realidad, confirmada por la coincidencia de todas las fuentes europeas, a lo largo de la época de la imprenta manual» ${ }^{9}$. O lo que es lo mismo: el pliego suelto -en sus diversas combinaciones de extensión y formato- es la unidad mínima de trabajo de la imprenta manual y solamente el plegado y la presencia de tinta diferencian un pliego de estas características y la hoja de papel, que por lo demás mantiene intacta su materialidad original.

De ello se deriva también, claro está, que cualquier impreso que no corresponda a un pliego de papel, modifica necesariamente ese estado material original de la hoja. Esto ocurre, por ejemplo, con todos aquellos impresos que superan la extensión equivalente a una hoja doblada un número indeterminado de veces, desde el pliego suelto en cuarto de varias hojas -el cual, en contra de lo que su nombre parece indicar, deja de ser en propiedad un pliego- hasta aquellos impresos de varios cientos de hojas formados por cuadernos que, a su vez, se componen de pliegos, vayan estos conjugados o no. Y también ocurre, evidentemente, por abajo, con aquellos impresos para cuya obtención es necesario romper la unidad material que constituye la hoja, cortándola después de impresa. Aquellas obras cuya extensión era menor al espacio tipográfico que ofrece un pliego natural, por tanto, podían compartir forma con otras de longitud igualmente reducida, de modo que en principio son posibles tantas combinaciones como permita el dicho papel de marca: «en un único pliego podían incorporarse, por ejemplo, dos impresos de medio pliego, o tres impresos, uno de medio pliego y dos de un cuarto de pliego o cuatro impresos de un cuarto de pliego ${ }^{10}$.

Estos impresos menores -adjetivo con ello únicamente su extensiónplantean, sin embargo, ciertos interrogantes, puesto que si bien por un lado

\footnotetext{
9 Jaime Moll, «El taller de la imprenta», en Historia de la edición y de la lectura en España, 14721914, ed. de Víctor Infantes, François Lopez y Jean-François Botrel, Madrid, Fundación Germán Sánchez Ruipérez, 2003, pp. 31-38.

${ }^{10}$ Manuel Pedraza García, «La Bula del Santo Sepulcro en su proceso criminal de predicación de bula falsa: una buleta impresa desconocida zaragozana del siglo xvI», en Gutemberg Jahrbuch, 84 (2009), pp. 184-202, p. 188.
} 
ofrecen el acomodo justo para ciertos textos, por otro implican más trabajo que el pliego natural, ya que hay que cortarlos, creándose así una tensión de intereses. De ahí que el objetivo de este trabajo sea indagar en los modos en que los talleres de imprenta abordaron esta situación y cuáles fueron las soluciones prácticas que se adoptaron. Aquí voy a centrarme en los impresos bifolios derivados de un pliego en cuarto de cuatro hojas. Para ello, comenzaré tratando algunas cuestiones técnicas sobre su producción, para, a continuación, rastrear algunos de estos impresos en volúmenes facticios y colecciones antiguas, de lo que se desprenden una serie de conclusiones que permiten dar algunas respuestas a estos problemas y redundan en una mejor comprensión de la transmisión de este tipo de impresos.

\section{De la hoja al bifolio: técnicas y problemas}

Cuando en la actualidad se habla de pliegos bifolios, la crítica suele referirse a impresos de dos hojas. Esta apreciación pudiera parecer innecesaria por evidente, pero esconde un problema notable: ¿cuáles son los requisitos formales para considerar a un impreso como bifolio? ¿basta que un impreso tenga dos hojas para considerarlo como tal o es necesaria la conjugación de varios factores? Para entender a lo que me refiero, es necesario que veamos cuáles eran las posibilidades técnicas de producción de estos impresos.

Como ya hemos dicho, un pliego de dos hojas -de no mencionar explícitamente lo contrario, nos referimos siempre a pliegos en cuarto- resulta de cortar un pliego de cuatro impreso previamente por las dos caras, de modo que la obtención de un impreso de dos hojas pasa necesariamente por la impresión previa de uno de cuatro. Dicho de otro modo: mientras un pliego de cuatro hojas puede entenderse, en su vertiente material, como el resultado directo del proceso de impresión, el pliego bifolio se obtiene manualmente a partir de este, por lo que sus características materiales y editoriales deben planearse con antelación. En general, puede suponerse que cada uno de estos impresos menores comparte una serie de rasgos editoriales con pliegos más extensos, tales como una portada con título en tipografía distinta a la del cuerpo del texto y frecuentemente un grabado, es decir, aquellos elementos que Norton y Wilson, según hemos visto, consideraron típicos del pliego, pero aplicados a un impreso de menor extensión. De modo que estos elementos paratextuales son, a la postre, los que suelen inducir a la identificación del impreso como ente independiente.

Al impresor que desee imprimir dos pliegos bifolios en una única hoja se le plantean por tanto tres escenarios distintos: puede suceder bien que en 
esa hoja quieran imprimirse dos obras idénticas, que quieran imprimirse dos obras distintas pero con el mismo grabado, o que esas dos obras sean distintas y lleven grabados distintos (o una de ellas, o incluso ambas, carezca de grabados, lo que para nuestro caso es lo mismo). Esto es importante porque, en principio -aunque nunca estaremos a salvo de excepciones- cada uno de estos escenarios exige técnicamente una distinta imposición de las planas.

En el último de los casos citados, si las dos obras a imprimir son completamente distintas, la imposición puede ser la misma que para un pliego de cuatro hojas convencional: 4-1-5-8 en la forma exterior y 2-3-7-6 en la interior, de modo que la primera portada va en la primera plana y la segunda en la 5. Así, las dos portadas aparecen en la forma exterior, pero dado que los grabados son distintos (o solamente hay un grabado, o no hay ninguno), esto no supone un problema y el pliego, una vez impreso, da lugar a dos bifolios correlativos.

Sin embargo, si en ambos pliegos el grabado es el mismo -ya sea porque se imprimen dos bifolios iguales o porque, por el motivo que sea, pretenden usarse en ambas obras los mismos elementos tipográficos, tales como orlas o grabados- las planas no pueden imponerse del mismo modo ya que, obviamente, un mismo grabado no puede aparecer dos veces en la misma cara del pliego. Para solventar este problema, hay dos tipos distintos de imposición ${ }^{11}$.

El primero de ellos es el que la bibliografía anglosajona denomina halfsheet imposition by work and turn. En él, ambas caras de la hoja se imprimen con una sola forma en la que la distribución de las planas es, 4-3-1-2. En el otro caso, la imposición por medios pliegos da lugar igualmente a dos bifolios aunque, en este caso, la impresión de cada cara de la hoja se realiza con una forma distinta, de modo que la distribución de las planas sería como sigue: la forma externa contendría 4-7-1-6 y la interna 8-3-5-2. En ambos casos, a diferencia de lo que ocurre al plegar un cuarto según la primera de las imposiciones expuestas, los dos bifolios no aparecen correlativos tras el plegado, sino que uno queda abrazado por el otro ya que la paginación resultante sería

\footnotetext{
${ }^{11}$ Uno de los primeros trabajos en tratar este tipo de imposiciones fue el de Ronald McKerrow, $A n$ introduction to bibliography for literary students, Oxford, Clarendon Press, 1927, en el apartado «Halfsheet imposition», pp. 66-70. Al referirse a los orígenes de estas imposiciones, McKerrow se limita a mencionar prudentemente que esta «came into use at a period no easy to determine» (p. 66), y, centrándose en la imposición por medios pliegos en octavo, que esta se generaliza a partir del siglo xviii y que en su opinión «it is [...] comparatively rare in earlier times» (p.67). Posteriormente, K. Povey, «On the Diagnosis of Half-Sheet Impositions», en The Library, 5 (1956), pp. 268-272, mostró que el uso de estas imposiciones es bastante anterior. Hoy sabemos que fue común incluso desde la época incunable. Una descripción detallada, con gráficas explicativas, en Philip Gaskell, A New Introduction to Bibliography, Oxford, Clarendon Press, 1972, especialmente p. 42 y p. 83 así como las figuras 48, 49, 52, 53, 58 y 59. Para casos concretos en el ámbito hispánico, pueden verse los trabajos de Mercedes Fernández Valladares citados en las notas siguientes.
} 
1, 2-5, 6, 7, 8-3, 4. Separando ese pliego interno de las hojas que lo abrazan obtendríamos dos pliegos bifolios: A $(1,2-3,4)$ y B $(5,6-7,8)$.

Un ejemplo precioso de este último tipo de imposición lo constituyen (o constituyeron) los pliegos RM476 $6^{12}$ y RM605 $5^{13}$, las Coplas del huevo y la Copla que hizo tremar respectivamente, impresos según Mercedes Fernández Valladares en Burgos, por Alonso de Melgar, «c.1521-22 [ant. mayo]» ${ }^{14}$. Tras rastrear su historia y sus particularidades tipográficas, Fernández Valladares ha demostrado que la imposición utilizada para esos pliegos fue la indicada: «la figurilla de la dama contigua a la casa [...] al aparecer repetida en la portada de Siguense vnas coplas del huevo y en la portadilla de la Copla que hizo tremar a vna alcahueta -mostrando además en ambos casos una grieta en la parte derecha de la frente-, se demuestra que es el mismo taco y, por tanto, que esas planas forzosamente tuvieron que imponerse cada una en una forma distinta del pliego, es decir, una en el blanco y otra en la retiración, tratándose por ello de un caso claro de imposición por medios pliegos» ${ }^{15}$. Nos encontramos, por tanto, frente a:

dos pliegos de dos hojas cada uno, que fueron impuestos simultáneamente e impresos en una misma tirada, pero destinados a una distribución comercial independiente. Es decir, aunque como producto tipográfico las dos composiciones se imprimieron como una unidad, ocupando cada una dos hojas -de las cuatro del pliego de papel-, como producto editorial se planificó la imposición de sus moldes de tal modo que el resultado fueran dos piezas independientes -dos «pliegos sueltos»-, obtenidos tras dividir el pliego de papel por el lado corto una vez impreso ${ }^{16}$.

Como vemos, Fernández Valladares recurre para arrojar luz sobre el fenómeno a una distinción terminológica que toma de Julián Martín Abad, la diferencia entre producto tipográfico y producto editorial ${ }^{17}$. Hemos de notar, con

${ }^{12}$ [En línea]. Enlace: <http://data.onb.ac.at/rep/1086AA2D> [Consulta: 11/12/2019].

${ }^{13}$ Hay edición facsímil en Pliegos poéticos góticos de la Biblioteca Nacional de Madrid, introducción de José Antonio García Noblejas, Madrid, Joyas Bibliográficas, 1957-1961, vol. II, n. 77.

${ }^{14}$ Mercedes Fernández Valladares, La imprenta en Burgos (1501-1600), Madrid, Arco Libros, 2005, n. ${ }^{0} 136$.

${ }^{15}$ Fernández Valladares, art. cit., p. 102. Véase también Mercedes Fernández Valladares y Francisco Mendoza Díaz-Maroto, «Los impresos burgaleses de don Hernando Colón: algunas concordancias y rastros de pliegos sueltos colombinos (con la noticia de una nueva edición del cuentecillo del rústico labrador)», en Geh hin und lehre. Homenaje a Klaus Wagner, ed. de Piedad Bolaños, Sevilla, Secretariado de publicaciones de la Universidad de Sevilla, 2007, pp. 193-228, especialmente p. 219, n. 107.

${ }^{16}$ Fernández Valladares, art. cit, pp. 100-101

${ }^{17}$ Julián Martín Abad, Los libros impresos antiguos, Valladolid, Universidad de Valladolid, 2004, especialmente capítulo 2, «El producto tipográfico», pp. 25-60 y capítulo 3, «El producto editorial», pp. 61-87. 
todo, que en principio estos dos conceptos no designan entidades diferentes, sino que, al igual que sucede con los conceptos de producto textual, producto histórico y producto bibliográfico, son usados por Martín Abad para diferenciar distintas perspectivas con las que abordar el impreso: basta comparar el inicio del capítulo que dedica a los primeros - «cada ejemplar es un producto tipográfico» ${ }^{18}$ - con el que dedica a los segundos - «cada ejemplar de una edición antigua es además un producto editorial» ${ }^{19}$ - para constatarlo. Dado que el producto tipográfico puede identificarse como el resultado de «tres operaciones sucesivas: la composición, el casado y la imposición, y la tirada ${ }^{20}$, no parece haber inconveniente en contemplar un pliego de cuatro hojas como tal, independientemente de que pueda contener una o más obras; sin embargo, la condición de producto editorial, aunque se infiere de la presencia de una serie de elementos concretos - de la portada al colofón, pasando por frontispicios, preliminares, etc., sin que tengan que estar todos ellos presentes ${ }^{21}-$ me parece bastante más difícil de atribuir.

Aunque parece evidente que para poder considerar, desde una perspectiva editorial, un bifolio como tal, este debe constar materialmente de dos hojas, lo que en realidad resulta verdaderamente determinante es que esas dos hojas sean la consecuencia de la estrategia editorial concebida para la obra en cuestión. Son obvios los motivos por los que suele asociarse la condición bifolia a un estado material concreto como son las dos hojas, pero en muchas ocasiones, esa condición se ha otorgado al impreso partiendo únicamente de un estado material concreto y la presencia en esa materialidad de los elementos de los que solemos inferir que estamos frente a un producto editorial. Muy pocas veces, sin embargo, estamos en condiciones de afirmar, frente a un pliego de dos hojas, que ese estado material sea resultado de la voluntad editorial. O a la inversa: no siempre podemos afirmar, frente a un pliego de cuatro hojas, si se compone de dos bifolios o no.

En el ejemplo que acabamos de ver, así como en aquellas imposiciones para dos ejemplares iguales, el cortado se antoja imprescindible, pues de otro modo la lectura de uno de los dos bifolios -del que «abraza»- quedaría interrumpida por el otro bifolio. Sin embargo, en el caso de la primera imposición el resultado es un pliego que puede ser leído como dos bifolios o como pliego en cuarto, de modo que el problema que se plantea es, aun cuando en esas

\footnotetext{
${ }^{18}$ Martín Abad, ob. cit. p. 25. La cursiva es del autor.

${ }^{19}$ Ibidem, p. 61.

${ }^{20}$ Ibidem, p. 25.

${ }^{21}$ Así parece comprenderlo Martín Abad, ob. cit., pp. 62-80, pues son estos los elementos que lista en el capítulo dedicado a este tipo de productos, sin que haya encontrado en esas páginas una definición más concreta.
} 
hojas encontremos los elementos característicos de un producto editorial, si disponemos de argumentos suficientes para considerar que realmente ese era el uso destinado para el impreso. Para complicar más las cosas, un análisis detallado de la historia de esos bifolios arroja resultados cuanto menos sorprendentes, ya que muchas veces -e independientemente de la imposición utilizada- los supuestos bifolios han adquirido esa materialidad en fechas relativamente recientes o simplemente no la han adquirido, lo que dificulta mucho poder considerarla como parte de su estrategia editorial.

\section{De pliegos abrazados y bifolios de cuatro hojas}

Los dos pliegos bifolios del ejemplo que acabamos de ver, RM476 y RM605, se conservan en la Österreichische Nationalbibliothek de Viena y en la Biblioteca Nacional de Madrid respectivamente. Sin embargo, como ya demostraron Askins e Infantes ${ }^{22}$, estos pliegos no se separaron en dos unidades tipográficas distintas hasta fechas muy avanzadas en relación a su fecha de impresión. En el catálogo de la biblioteca de Richard Heber ${ }^{23}$, uno de sus antiguos poseedores, ambos pliegos aún formaban un único producto tipográfico, pero cuando la colección salió a subasta estaba claro que las ganancias de la venta «podrían multiplicarse si las piezas se ofrecían por separado» ${ }^{24}$. Y así, en efecto, en el Catalogue de la biblioteca de Van Berghem, que las adquirió de Heber, las piezas ya aparecen por separado ${ }^{25}$ y así se mantienen hasta la actualidad. Mercedes Fernández Valladares y Francisco Mendoza rastrearon los orígenes del pliego - ¿de los pliegos?- llegando hasta su primer poseedor, Hernando Colón, a quién los dos bifolios le fueron vendidos como una única unidad. Los citados investigadores parecen creer que la presencia en los anaqueles del bibliófilo sevillano de este pliego sin separar se debe a un cúmulo de circunstancias accidentales. Así, escriben, el pliego «le fue vendido curiosamente sin cortar y en esta forma ingresó y permaneció en su

\footnotetext{
${ }^{22}$ Arthur L. F. Askins y Víctor Infantes, «Las "Coplas” celestinescas de ¿Tremar? Una historia casi completa de medio pliego», en Celestinesca, 15 (1991), pp. 31-51.

${ }^{23}$ Bibliotheca Heberiana. Catalogue of the Library of the late Richard Heber, Pall Mall, W.Nicoi, 1835. Sobre la venta de esta colección véase el trabajo de Arnold Hunt, "The Sale of Richard Hebers Library», en Under the hammer: book auctions since the seventeenth century, ed. de Michael Harris, Giles Mandelbrote y Robin Meyers, London, Oak Knoll Press, 2001, pp. 143-171.

${ }^{24}$ Askins e Infantes, art. cit., p. 46.

${ }^{25}$ Catalogue de la première partie des livres composant la bibliothèque de feu M. Van Berghem, París, Maison Silvestre, 1836. Curiosamente, Askins e Infantes, art. cit., p. 46, indican que en este catálogo aparecen las piezas de Heber «ya sin el mismo orden que poseían [allí] y entre ellas nuestros pliegos, todavía uno a continuación del otro y con la mención de "2 feuill”». En realidad, ya están separadas, siendo las Coplas que hablan de como las mugeres... el número 484 y las de tremar el 487.
} 
biblioteca, bien por prurito bibliofílico o más seguramente por no reparar en que, en realidad, contenía dos piezas independientes impresas a la vez» ${ }^{26}$, según parece sugerir «el desorden apreciable en la notación de los íncipits y éxplicits respectivos de cada pieza en el registro colombino» ${ }^{27}$.

Ahora bien, ¿con qué argumentos podemos afirmar que estamos ante una circunstancia accidental? Hasta donde llegan mis conocimientos, muy pocas veces podemos decir de un bifolio - de un ejemplar de dos hojas- que ese fue el estado material en que se distribuyó originalmente ${ }^{28}$, pero abundan sospechosamente, en cambio, aquellos casos en los que los supuestos bifolios han llegado hasta nosotros unidos o aquellos en los que podemos demostrar que, al igual que el ejemplo mencionado, se separaron tardíamente, por motivos que nada tienen que ver con la intención editorial original con que se imprimió la obra.

Como ejemplo de pliegos bifolios que han llegado unidos hasta nosotros resulta ejemplar el caso de una de las colecciones de pliegos más recientemente descubiertas, el volumen Ant.Il.1402 de la Biblioteca Comunale Augusta de Perugia. Al describirlo, Víctor Infantes apuntó: «se conserva con una encuadernación en pergamino completo flexible muy sucio, ajado y con esporulaciones, con restos de dos cierres de cintas, tres costuras en pergamino y doble contratapa de vuelta -ha perdido una de las de la cubierta delantera- y es original de la época de los impresos, lo que demuestra que la colección se encuadernó a la vez, sin tener en cuenta que se incluían pliegos repetidos» ${ }^{29}$. Esa repetición, sin embargo, no significa únicamente que un pliego aparezca más de una vez dentro del volumen, sino, en muchos casos, que un pliego de cuatro hojas conteniendo dos ejemplares iguales de un mismo pliego ha sido encuadernado sin cortar, es decir, exactamente en el mismo estado en que se encontraban originalmente las Coplas del huevo y la Copla que hizo tremar como veíamos más arriba.

Ya sabemos que cuando se querían obtener dos ejemplares idénticos el pliego se imprimía con una única forma, de modo que, al no cortarlo, al 1v del que debería ser el primer pliego bifolio le sigue $1 \mathrm{r}$ del segundo pliego

\footnotetext{
${ }^{26}$ Así lo sugiere Fernández Valladares, art. cit., p. 101.

${ }^{27}$ Fernández Valladares y Mendoza Díaz-Maroto, art. cit., n. 107.

${ }^{28}$ Téngase en cuenta, además, la imposibilidad en la mayoría de ocasiones de demostrar materialmente que dos pliegos se imprimieron juntos, por lo que para ello debemos recurrir a otro tipo de indicios. Además de los trabajos citados arriba, véanse también las útiles aclaraciones de Stanley Boorman, «A Case of Work and Turn Half-Sheet Imposition in the Early Sixteenh Century», en The Library, 8:4 (1986), pp. 301-321.

${ }^{29}$ Víctor Infantes, «Una cuarentena poética desconocida. Los pliegos sueltos del siglo XVI de la Biblioteca Comunale Augusta de Perugia», en Criticon, 117 (2013), pp. 29-63, p. 29.
} 
bifolio, a este el de la segunda hoja del primer bifolio y, finalmente, la segunda del segundo. Víctor Infantes identificó los pliegos en cuestión como bifolios partiendo de una serie de elementos externos, editoriales, pero creyó que el estado en que se encontraban en el volumen era fruto de una mala encuadernación. Así, al hablar de dos de los bifolios del volumen que él creía repetidos -en el sentido mencionado- escribió: «en el mismo volumen, a continuación [del número 7], se encuentra otro ejemplar del mismo pliego, n. ${ }^{\circ} 7$ (bis), pero al encuadernarlo se ha embuchado uno en el otro, con lo que se ha trastocado el orden y ahora es: h. $1+$ h. $1+$ h. $2+$ h. $2 »^{30}$. En realidad, con estas Cobles ara nouament trobades, a llaor y gloria del sanctissim cos precios de Jesu Christ, impresas por Juan Timoneda -RM555.5, números 7 y 7 (bis) de la colección- lo que ha sucedido es obviamente que un único pliego de cuatro hojas con dos bifolios se ha encuadernado sin haber sido cortado antes.

No son los únicos casos que encontramos en que esto ocurre: al contrario, hasta cuatro más podemos hallar en ese mismo volumen. Se da la misma situación con los números 12 y 12(bis), dos ejemplares de Los goigs d [e] la verge Maria / del Socors. Ab los goigs dels gloriosos metges, sent Cosme, e sent Damia. Joan Mahiques, que ha estudiado numerosos aspectos del volumen, también creyó que se trataba de un ejemplar «descompaginat, car té un foli abans i l'altre foli després del següent exemplar» ${ }^{31}$. Como vemos, se trata de la misma particularidad que el pliego anterior, dos primeras páginas, luego la segunda del segundo cuaderno y finalmente la segunda del primero, fruto de la imposición utilizada. Otro caso: el número 21, Siguen se tres canciones muy / sentidas y regozigadas para cantar la noche de $\mathrm{Na}=/$ uidad. Compuestas por Ausias Yzquierdo. / Año de. M.D. Lxj, impreso en Valencia por Joan Navarro en ese 1561, también va abrazando otro ejemplar igual, 21(bis) (RM264.8). Los dos siguientes, 22 y 22bis (RM953.3), son igualmente dos ejemplares en esa disposición tipográfica de la Obra en la qual se tracta de cómo Judas vendio a Christo, de la que también hay un ejemplar suelto en la misma colección (n..$^{\circ} 11$ ). Y sucede, finalmente, lo mismo con los dos siguientes, otros gozos, en este caso Los goigs de la sacratissi=/ ma verge Maria de Lorito. Ab los goigs de la / verge Maria del Roser. Y los goigs de /

${ }^{30} \mathrm{Ibidem}$. Así ha pasado al Suplemento, ob. cit., donde bajo la misma entrada, 555.5, se registran los dos ejemplares, 7 y 7(bis) y se anota la cita que aquí reproducimos.

${ }^{31}$ Joan Mahiques, «Goigs valencians en dos plecs poètics de la Biblioteca Comunale Augusta de Perugia», en Estudis de Llengua i Literatura Catalanes. Miscel-lània Jordi Bruguera, I, Barcelona, Publicacions de l'Abadia de Montserrat, 2013, pp. 23-37, p. 27. Quisiera agradecer expresamente a Joan Mahiques que me proporcionara copia del volumen de Perugia. 
sant Uicente Ferrer, por el mismo impresor y en el mismo año de 1561 que el ejemplo anterior.

Partiendo de esta tipología, creo que es posible aclarar otros casos anómalos, algo más complejos, que encontramos en este mismo volumen. En las primeras páginas, por ejemplo, encontramos repetidas las dos primeras hojas de un mismo pliego, las Coplas de si me vieras Juan (RM793.5, $\mathrm{n}^{\text {os }} 1$ y $1+\mathrm{ev}$ según la numeración de la Cuarentena). Víctor Infantes anotó para estas dos hojas que se trataba de dos ejemplares fragmentarios, de los cuales «sólo se conserva la primera hoja, que contiene parte de la primera composición $\rangle^{32}$, y en parte llevaba toda la razón, pero solo en parte. Gracias al pliego número 6 del mismo volumen -otro ejemplar del pliego, que en este caso se conserva «entero»- sabemos que esta hoja representa la primera de un bifolio. Con ello, esas dos primeras hojas serían también las dos primeras hojas de un pliego sin cortar, de una hoja entera que, plegada, daba lugar a dos bifolios, uno de los cuales abrazaba al otro. Al describir el tercero de los pliegos de la colección - no 1(bis), RM1179.5-, Infantes apuntó también que «entre la hoja final del pliego anterior y la primera del pliego siguiente se observa la existencia de 2 hojas, lo que nos hace suponer que su constitución original era de 4 hojas, conservando en la actualidad solamente las dos últimas $\rangle^{33}$; en realidad, entre las dos hojas sueltas y las conservadas de RM1179.5 se conservan restos de varias hojas - parecen cuatro- de modo que podemos creer que esos restos no solamente corresponderían a las primeras de 1(bis), sino también a las dos segundas hojas de los respectivos bifolios o, si se quiere, a la tercera y cuarta hoja del pliego sin cortar del cual conservamos -registrados como 1 y $1+$ ev- las dos primeras primeras hojas.

Que según acabamos de ver en algunos casos haya hasta tres ejemplares de un mismo pliego -dos en un pliego de cuatro hojas y otro suelto, como sucede por ejemplo con 22, 22bis y 11- hace suponer que probablemente RodríguezMoñino ${ }^{34}$ y Víctor Infantes ${ }^{35}$ llevaran razón al creer que este tipo de facticios -ocurre algo similar en otras colecciones, como veremos más abajo- podrían

\footnotetext{
${ }^{32}$ Idem, n. ${ }^{\circ} 1 \mathrm{ev}$.

${ }^{33}$ Infantes, «Cuarentena...», art. cit., p. 33, n. ${ }^{\circ} 1$.

${ }^{34}$ Antonio Rodríguez-Moñino, «Los pliegos poéticos de la colección Campo de Alanje en la Biblioteca Nacional de Madrid (siglo XVI)», en Romance Philology, 17 (1963), pp. 373-380, «...casi siempre todo el contenido del volumen es contemporáneo, porque hay que suponer que se trata del fondo existente en las manos de un vendedor del siglo XVI», p. 374. Y nótese que también apuntó que «a veces en uno de estos facticios hay dos ejemplares exactamente iguales del mismo pliego», p. 376.

35 «Esta cercanía temporal de las fechas de impresión sugiere -como hemos indicado- la posible adquisición en algún librero que poseyera un surtido de pliegos sueltos de relativa novedad editorial -lo que explicaría los duplicados y la mezcla de verso y prosa- y no una selección cuidadosa con criterios específicos», Infantes, «Cuarentena...», art. cit., p. 30.
} 
representar los fondos de algún vendedor contemporáneo de pliegos, aunque de hecho -al menos en mi opinión- esto no ofrece una explicación para el fenómeno. Excluida ya la posibilidad de que se trate de un error de encuadernación, ¿por qué habrían de llevar los vendedores este tipo de productos sin cortar?, ¿se adquirieron acaso estos lotes en un estadio material previo a la venta al público? No podemos dar aún respuesta a estas preguntas, pero lo cierto es que, una vez constatado que el caso de Colón no es algo aislado, ciertos casos particulares en la transmisión parecen menos extraños.

Así, por ejemplo, he podido notar que, entre los pliegos sueltos poéticos hasta 1600 -campo donde sabemos que las pérdidas deben de haber sido inmensas y en el que de la mayoría de ellos conocemos un único ejemplar-, la presencia de ejemplares dobles entre los bifolios es especialmente alta. Partiendo de un total aproximado de unos 1110 pliegos $^{36}$, he contado 103 pliegos registrados como bifolios, lo que supone que tendríamos por un lado estos 103 impresos y por otro los 1007 pliegos restantes, todos ellos de distintos formatos y extensiones, del folio al doceavo y de la hoja suelta a las 20 páginas. Pues bien, de entre esos 1007 pliegos, conocemos la existencia de más de un ejemplar para 27 de ellos, lo que equivale -insisto en la provisionalidad de estos cálculos- a un 2,7\% de los pliegos que no son bifolios en cuarto. Entre los 103 bifolios, en cambio, de 11 se conservan dos ejemplares, lo que equivale a un $11,3 \%$, cuatro veces más, por tanto, que entre los de más extensión. Este hecho, además, ni tan solo es particular de la época estudiada, sino también constatable en épocas posteriores ${ }^{37}$.

¿A qué se debe que el número de ejemplares dobles sea notoriamente elevado entre los pliegos bifolios? A la luz de lo expuesto creo que la explicación más verosímil es que, al igual que ocurre con los casos de Perugia o con el pliego colombino, muchos de ellos no se separaran en dos unidades independientes hasta una fase relativamente reciente de su transmisión, lo que favoreció su conservación. Todo indica, por el momento, que al contrario de

\footnotetext{
${ }^{36}$ Aproximado porque no siempre queda claro si un pliego de dos hojas es realmente bifolio o fragmentario o también porque algunos ejemplares dobles se cuentan como entradas independientes. No he tenido en cuenta, para este cómputo, las series valencianas del romancero nuevo.

${ }^{37}$ Dejemos anotado, como ejemplo en favor de esta tendencia, el caso de los pliegos del viajero inglés Samuel Pepys, sobre cuya colección deben consultarse los trabajos de Edward Wilson, «Samuel Pepys's Spanish Chap-Books, Part I», Transactions of the Cambridge Bibliographical Society, 2 (1955), 2, pp. 127-154 y «Samuel Pepys's Spanish Chap-Books, Part II», Transactions of the Cambridge Bibliographical Society, 3 (1956), pp. 229-268. Pepys adquirió su colección de pliegos «in Cadiz and Seville during the Winter of 1683/84» I, p. 130. De los 75 pliegos que la componen, hay uno que aparentemente «is duplicated», II, p. 127 ; sin embargo, no es propiamente un pliego repetido ni un duplicado, sino un único pliego con dos ejemplares iguales, bifolios, de las Letras de los villancicos que se cantaron en la Santa Iglesia Cathedral de Cadiz, números 28 y 29 de la colección de Pepys
} 
lo que pudiera sugerir la lógica, muchos bifolios se distribuyeron sin separar, al menos en una primera instancia.

$\mathrm{Y}$ en efecto, si buceamos por la historia de las diversas manos por que han pasado algunos de los ejemplares conocidos podemos constatar que, en muchas ocasiones, en aquellos volúmenes que reúnen (reunían) las condiciones adecuadas, esto es, ser contemporáneos de los materiales que contiene, estos bifolios se hallaban contigüos a otros bifolios, lo que -conociendo los precedentes- interpreto como indicio de que pudieran constituir un único producto, al menos tipográfico. No hay que olvidar que casos como el del volumen facticio de Perugia no dejan de ser atípicos, pues muchos volúmenes que respondían a esos criterios se desmembraron, especialmente en el siglo XIX y a principios del xx. En algunos casos, sin embargo, es posible reconstruir su estado original.

\section{¿Pliegos de cuatro hojas o bifolios contiguos?}

Uno de los casos en que esto ocurre es el de la colección que perteneció a la Condesa de Campo de Alanje, conservada hoy -en un orden que no se corresponde al original- en la Biblioteca Nacional de Madrid. Como escribió Antonio Rodríguez-Moñino, «constituye el volumen procedente de la colección de Campo de Alanje, uno de los más importantes núcleos de la Biblioteca Nacional de Madrid, pero, desgraciadamente, en vez de conservar la unidad de procedencia, fueron caprichosamente desglosados y ni siquiera se hallan en los estantes con una situación correlativa ${ }^{38}$. Por suerte, el insigne bibliógrafo pudo reconstruir el orden del volumen original gracias a un Catálogo de las obras procedentes de la Biblioteca de la Condesa de Campo de Alange recibidas en la Biblioteca Nacional, manuscrito, conservado hoy en la Hispanic Society de Nueva York. Así, sabemos que este de la condesa era «un volumen en $4^{\circ}$, encuadernado en becerro, con adornos estampados de la época, es decir, del siglo XVI, en su primera mitad ${ }^{39}$, por lo que asumimos que hasta su separación, el estado y orden de las obras allí contenidas respondía al de su colecta. De las 56 obras que componían el volumen, solamente seis son pliegos bifolios, pero precisamente esas seis van agrupadas en tres

${ }^{38}$ Rodríguez-Moñino, art. cit., p. 376.

${ }^{39}$ Ibidem. 
grupos de dos pliegos, en los números 28 y $29\left(\mathrm{RM} 665^{40}\right.$ y RM310 $\left.{ }^{41}\right), 33$ y $34\left(\mathrm{RM} 1064^{42}\right.$ y RM423 ${ }^{43}$ ) y 45 y 46 (RM1062 $2^{44}$ y RM705 ${ }^{45}$ ).

No podemos afirmar que esta agrupación supusiera una unión de los dos bifolios en el caso de RM1064 y RM423 (33 y 34 en el volumen de Campo de Alanje). El primero de ellos, titulado Siguense dos romances de Gayferos, va decorado con cuatro figuritas enmarcadas en una orla de cuatro barras, mientras que el segundo, un Romance del conde claros nueuamente trobado por otra manera. Fecho por Anton Pansac Andaluz, lleva igualmente una orla de cuatro barras, y dentro una figurita suelta a izquierda y un grabado de dos jinetes a la derecha de esta. Como lamentablemente no disponemos de un estudio tipográfico detenido de estos pliegos, ni siquiera podemos afirmar que procedan del mismo taller, requisito imprescindible para tener en consideración la hipótesis de que constituyeran un único pliego de cuatro hojas. Muy distintos son, sin embargo, los otros dos casos, ya que para ellos sí disponemos de datos concretos que nos permiten profundizar más.

En el primero de ellos, los dos pliegos en cuestión, RM665 y RM310 (28 y 29), han sido adscritos ambos a la misma imprenta, la burgalesa de Juan de Junta, y datados $c .1538-40$ el primero ${ }^{46}$ y $c .1535-40$ el segundo ${ }^{47}$, por lo que disponemos de una base sólida sobre la que exponer nuestras sospechas. El primero de ellos lleva por título Aqui comiençan las coplas de Madalenic (sic) Con otras coplas de la reyna de Napoles. Con vna cancion, mientras que el segundo se titula Coplas nueuamente hechas por Francisco de Lora a este Villancico que dize. Mariquita fue a la plaza mas ha de vn hora no puede mas la peccadora. Con vna glosa del mismo lora a las coplas de Desamada siempre seas: ames y nunca te amen. E otras dos maneras de coplas.

Nótese que el segundo de los pliegos menciona al final del título dos maneras de coplas, pero estas no aparecen luego en el pliego, lo que llevó a Rodríguez-Moñino a suponer que el pliego estaba incompleto. No es el único pliego que conocemos con esa particularidad. El propio Rodríguez-Moñino notó que «igual ocurre con el ejemplar de una edición distinta que se conserva en el Museo Británico de Londres, el cual está en las mismas circunstancias»,

\footnotetext{
${ }^{40}$ Facsímil en Pliegos Madrid, ob. cit., III, n. ${ }^{0} 120$.

${ }^{41}$ Facsímil en Pliegos Madrid, ob. cit., III, n. ${ }^{\circ} 121$.

${ }^{42}$ Facsímil en Pliegos Madrid, ob. cit., IV, n. ${ }^{\circ} 162$.

${ }^{43}$ Facsímil en Pliegos Madrid, ob. cit., III, n. ${ }^{\circ} 126$.

${ }^{44}$ Facsímil en Pliegos Madrid, ob. cit., III, n. ${ }^{\circ}$ 125, editado anteriormente por Vicente Castañeda y Amalio Huarte, Nueva Colección de pliegos sueltos, Madrid, Tipografía de Archivos, 1933, n. ${ }^{\circ} 2$.

${ }^{45}$ Facsímil en Pliegos Madrid, ob. cit., III, n. ${ }^{\circ} 128$, anteriormente también editado por Vicente Castañeda y Amalio Huarte, Colección de pliegos sueltos, Madrid, Revista de Archivos, 1929, n. ${ }^{\circ} 30$.

${ }^{46}$ Fernández Valladares, ob. cit., n. ${ }^{\circ} 342$.

${ }^{47}$ Idem, n. ${ }^{\circ} 339$.
} 
por lo que supuso que «el uno copia al otro y ambos se derivan de un ejemplar que estaba falto por el final y que fue reproducido ciegamente por el primer impresor sin fijarse en que el contenido no correspondía al título» ${ }^{48}$. El pliego de la British Library al que se refiere Rodríguez-Moñino es el RM309 ${ }^{49}$, que efectivamente lleva el mismo título e igual contenido que nuestro RM310, es decir, parece hallarse falto por el final de esas dos maneras de coplas. Sin embargo, como ya notó Askins al prologar la edición facsimil de los pliegos poéticos de Londres, Hernando Colón, que poseyó este pliego, anotó en el asiento 15009 de su Abecedarium $B$ además de las composiciones que efectivamente encontramos en el pliego, «dos asientos para lo que serán las 'otras maneras de coplas': col.25 [Abrasme magdalenica ay jesus quien anda ay] y col. 542 [Emperatrices y reinas que huys del alegria] ${ }^{50}$. Y esas dos maneras de coplas son precisamente las que contiene el pliego RM665, el que le precede en el volumen de Campo de Alanje.

Pero hay más: el pliego de la British Libray, RM310, procede de la colección de Richard Heber, concretamente del volumen que en el catálogo de su biblioteca llevaba el número 2818 , dentro del cual ocupaba el número $4^{51}$. Y precisamente el número 5 era RM666, es decir, otra edición de las Coplas de madalenica. Hoy, este pliego, RM666, se conserva en la Hispanic Society de Nueva York y RM309 en Londres, pero en el volumen de Heber ambos iban contiguos, lo que demuestra que constituyen -materialmente hablando- un único pliego; es decir: no hubo un impresor que en algún momento de la cadena de transmisión no notó que el contenido no correspondía con el título, sino alguien que no comprendió que los supuestos bifolios no eran tales. Y con esto de paso se demuestra también que RM665 y RM310 (28 y 29 de Campo Alanje) son (eran) también un único pliego. Aunque el par RM309/ RM666, a diferencia de lo que sucede con RM310/RM665, no ha sido datado ni atribuido a una imprenta concreta, creo que resulta evidente que existe entre ellos una relación iconográfico-semántica directa o indirecta. No podemos precisar la dirección de esa dependencia, pero, más allá de los títulos, resulta imposible que la coincidencia prácticamente total en la posición de las diversas figuritas factotum que aparecen en portada sea casual.

Pero si se trata, como suponemos, de dos pliegos de cuatro hojas, hemos de solucionar otro problema, pues -frente a lo que ocurría en el volumen de

\footnotetext{
${ }^{48}$ Nuevo Diccionario, ob. cit., p. 330, RM310.

${ }^{49}$ Facsímil en Arthur L. F. Askins, Pliegos poéticos españoles de la British Library, Londres (impresos antes de 1601), Madrid, Joyas Bibliográficas, 1989-1991, n. ${ }^{\circ} 30$

${ }^{50}$ Idem, p. 93.

${ }^{51}$ Bibliotheca Heberiana, ob. cit.
} 
Heber- los dos bifolios de la colección de Campo Alanje aparecían en el volumen original en el orden inverso al esperable. Quizá la explicación pudiera ser tan simple como un mal plegado. En este caso, a diferencia de lo que sucedía en la mayoría de ejemplos del volumen de Perugia, nos las habemos con dos bifolios distintos en los que, además, no se ha usado ningún grabado común, de modo que puede imprimirse partiendo de una imposición corriente en cuarto. En ella tenemos frente a nosotros la cara externa, con las planas -de arriba a abajo, leyéndolas de izquierda a derecha- 8,5,1,452. Para obtener el cuaderno que deseamos hemos de doblar primero por el lado corto, es decir, desde nuestra vertical. Ahora podríamos tener frente a nosotros las planas 4 y 5 , que habrían de doblarse de modo que 4 quede encima de 5 . Si, por el contrario, dejamos 5 encima y doblamos 4 hacia atrás el segundo bifolio pasa a ser el primero y cuando llegamos al $2 \mathrm{v}$ (que en realidad es $4 \mathrm{v}$ ) comienza el otro bifolio. O lo que es lo mismo, aplicado a nuestro caso: RM310 y RM665 aparecerían en el orden invertido. No creo, sin embargo, que esto sea lo que sucedió en el volumen de Campo Alanje; se trata de una posibilidad teórica que debe tenerse en cuenta al analizar otros posibles casos, pero en el que nos ocupa se da la circunstancia de que en el par de bifolios que nos falta por analizar ocurre exactamente lo mismo, pues también están en el orden inverso al esperable.

Me refiero a los números 45 y 46 según el orden del volumen de Campo Alanje, RM1062 y RM705 en la numeración del Nuevo Diccionario. El primero de ellos, RM1062 (45), es una edición distinta del pliego que ya veíamos en RM1064, de esta misma colección: Siguense dos Romances de Gayferos, en los quales se contiene como mataron a don Galuan. El otro, RM705 (46), se titula Aqui comiença un romance del conde Guarinos almirante de la mar: trata como lo catiuaron los moros. A pesar de que no conozco intentos modernos de datación y atribución del pliego ${ }^{53}$, Castañeda y Huarte ya albergaron la sospecha de que ambos pliegos pudieran constituir un único impreso de cuatro hojas y, al editar en facsímil el pliego RM1062, hacían la siguiente reflexión: «uno y otro fueron de Campo Alange, y por la coincidencia en tipos y grabados sospechamos si este será continuación de aquel, ya que, aun dentro del capricho de estos encabezamientos, aquel dice "Aqui comiença" y este "Siguense", y entre los dos forman un pliego, que

\footnotetext{
${ }^{52}$ Gaskell, ob. cit., fig. 47.

${ }^{53}$ Castañeda y Huarte, Nueva Colección, ob. cit., p. 10, lo supusieron impreso en Barcelona por Pedro Malo a fines del siglo XVI, opción que parece descartada ya que Montserrat Lamarca, La impremta a Barcelona (1501-1600), Barcelona, Generalitat de Catalunya, 2015, no lo incluye en su repertorio.
} 
luego al encuadernarse se separaron ( $\mathrm{sic})\rangle^{54}$. Téngase en cuenta, sin embargo, que al llevar el mismo grabado, si formaron un solo pliego, esto implicaría que debieron de imponerse por medios pliegos a fin de que el grabado pueda usarse tanto en la forma interna como en la externa. Como ya hemos visto, en estos casos el segundo bifolio queda abrazado por el primero, pero en el volumen de Campo Alanje estos dos pliegos aparecían encuadernados de forma consecutiva. Dado que la imposición lo impide, ese orden implica que el pliego fue cortado antes de encuadernarse. Ahora bien, ¿por qué se encuaderno en ese orden? y ¿qué tiene que ver este caso con el anterior?

Una posible explicación, que creo que no se ha tenido en cuenta hasta ahora, es que estos pliegos antes de ser encuadernados hubieran sido «cortados» para su lectura; entrecomillo la palabra porque con ella no me refiero a separar dos bifolios, sino a lo que parece haber sido una práctica relativamente extendida para la lectura de estos pliegos. Víctor Infantes, en su conocido y pionero trabajo sobre constitución tipográfica y contenido literario de estos impresos, se planteaba cómo podrían leerse: «¿Se plegaban? Obviamente sí, por más que apenas nos hayan llegado en su estado original. Lo que plantea una pregunta inmediata: ¿se leían así?, ¿cómo?, invito a leer (hoy) un pliego (plegado) y se observará fácilmente la incomodidad del intento; ¿se cortaban?, ¿se desplegaban en el doblez opuesto para (ir) facilitando su lectura, motivo quizá de su destrucción y pérdida?» ${ }^{55}$. Pliegos más recientes, como los decimonónicos de la colección de pliegos de cordel del Seminario de Bibliografía de la Universidad Complutense de Madrid, se conservan aún, según Patricia García Sánchez-Migallón, «en su mayoría barbados en tres cuartas partes y una última parte permanecía (sic) intonsa» ${ }^{56}$. Otra opción es, por supuesto, que estos pliegos hubieran sido separados previamente con un corte total y luego encuadernados juntos. Ambas posibilidades pudieron haberse dado, pero ahora nos interesan más los motivos que llevaron a ese orden y creo que hallamos una explicación plausible en la disposición de los elementos en el vuelto de las respectivas segundas hojas de ambos bifolios. Como sabemos, el orden «correcto» sería RM705+RM1062, pero ocurre que

\footnotetext{
${ }^{54}$ Castañeda y Huarte, Nueva colección, ob. cit., p.10

${ }^{55}$ Víctor Infantes, «Los pliegos sueltos poéticos: constitución tipográfica y contenido literario», en $E l$ libro antiguo espanol (Actas del primer coloquio internacional, Madrid, 18 al 20 de diciembre de 1986), ed. de María Luisa López-Vidriero y Pedro M. Cátedra, Salamanca, Universidad de Salamanca, 1988, pp. 237-248, p. 244.

${ }^{56}$ Patricia García Sánchez-Migallón, La colección de pliegos de cordel y literatura popular del Seminario de Bibliografia (UCM): aproximación a su estudio bibliográfico, Máster Universitario en Literatura Española, defendido 20/9/2014, Tutora: Mercedes Fernández Valladares, p. 44. [En línea]. Enlace: $<$ https://eprints.ucm.es/29968/> [Consulta: 11/12/2019].
} 
mientras en RM1062 la segunda hoja tiene el vuelto muy lleno de texto, en RM705 este aparece casi vacío, con solamente once versos en la columna de la izquierda y nueve versos y la palabra fin en la columna de la derecha. El pliego original, tal y como pude comprobar en la Biblioteca Nacional de Madrid, presenta al final una rúbrica con las iniciales C.A., que suponemos de la condesa, lo que indicaría que efectivamente este bifolio -independientemente de si estaba separado o cortado para su lectura el pliego de cuatro hojas-se comprendió como el segundo por ser el más vacío, aunque en realidad era el primero $^{57}$. Algo parecido debió ocurrir con RM310 y RM665, donde podemos constatar que el vuelto de la segunda hoja de RM310 termina con un Fin centrado y en una tipografía notablemente mayor que el resto, mientras que en el vuelto de la segunda hoja de RM665, fin aparece en la misma tipografía que el cuerpo del texto y debajo de la columna de la derecha, lo que induce al error de suponer que RM310 es el pliego final. Parece evidente, así, que quien ordenó el volumen se dejó llevar más por esos criterios visuales que por lo que indican los títulos.

\section{Recepción y transmisión antigua de los pliegos bifolios}

No me cabe la menor duda de que lo expuesto hasta aquí debe tener sus consecuencias para la catalogación de estos impresos. Sin embargo, lo que más me interesa en este trabajo es indagar en las consecuencias que estas particularidades pudieron tener en la transmisión del pliego suelto poético. Y es que no debemos pasar por alto que si encontramos este tipo de fenómenos en las colecciones antiguas, lo más probable es que esta también fuera la fenomenología con que se encontraban aquellos receptores que más condicionaron la posterior transmisión de los textos: los propios editores e impresores quinientistas.

Hasta donde sabemos, cuando un taller reproducía un pliego anterior fuera este suyo o ajeno- lo más común era intentar reproducir no solo el texto sino también el aspecto externo, lo que permite postular que lo más probable es que en estos casos el original de imprenta utilizado fuera directamente el pliego que servía de base. Partiendo de esta hipótesis, hemos de suponer que además de bifolios de dos hojas, llegarían a esos impresores tanto pliegos editorialmente concebidos como bifolios pero que materialmente constituían un único pliego de cuatro hojas, como pliegos de cuatro hojas que constituían un único producto editorial. Y en estos casos la comprensión

\footnotetext{
${ }^{57}$ Esta particularidad no aparece reproducida en el facsímil -retocado- de la edición de Joyas Bibliográficas, pero si la reprodujeron Castañeda y Huarte, Colección, ob. cit., p. 208.
} 
que el impresor-editor hiciera de ese ejemplar condicionaba la transmisión posterior.

Acabamos de ver el caso de los supuestos bifolios, RM1062 y RM705. El primero llevaba por título Siguense dos Romances de Gayferos, en los quales se contiene como mataron a don Galuan y el otro, RM705 Aqui comiença un romance del conde Guarinos almirante de la mar: trata como lo catiuaron los moros, aunque como hemos visto el orden de los pliegos debería haber sido a la inversa. Pues bien, conocemos también un pliego algo tardío ( $c$. $1564-1570^{58}$ ), impreso por Felipe de Junta en Burgos (RM706 ${ }^{59}$ ), que presenta los dos romances de Gayferos en forma conjunta con el romance de Guarinos: Aqui comiença vn romance del conde Guarinos Almirante de la mar: y trata como lo captiuaron los moros. Y otros dos romances de Gayferos: en los quales se contiene como mataron a don Galuan. Y vnas coplas hechas por Rodrigo de Reynosa. Este título es algo distinto al que sugerirían los dos títulos independientes, pero dentro del pliego, al vuelto de la segunda hoja, encontramos como título introductorio precisamente Siguense dos romance de Gayferos, en donde la formulación e incluso la tipografía empleada remiten inevitablemente a un impreso anterior donde este fuera el comienzo (del pliego o de la hoja). Desde esta perspectiva, todo parece indicar que este pliego burgalés se formó tomando como base cuatro hojas; es decir, en algún momento de la cadena de transmisión el impresor se sirvió de un impreso que comprendió como de cuatro hojas. De hecho, aunque modernamente se hayan catalogado como bifolios, sabiendo que lo que hoy conocemos como RM1062 se titula Siguense dos romances y que efectivamente esas hojas seguian a otras dos ¿pueden considerarse esas dos hojas como independientes? Los impresores quinientistas, al menos, no las consideraron así. Es más, gracias a esto podemos retroceder en el tiempo y ver que probablemente, el pliego - de cuatro hojas- se transmitió y comprendió así desde fechas muy tempranas.

A pesar de que el pliego burgalés es tardío, existe también un pliego, RM1063, impreso en Barcelona por Carles Amorós en torno a $1525^{60}$ y considerado hasta ahora bifolio, aunque en realidad lo único que de él se conserva es una única hoja, la primera, en la que encontramos ese título de Siguense dos romance de Gayferos $^{61}$. Dada la similitud -que no identidad- de este

\footnotetext{
${ }^{58} \mathrm{La}$ fecha la propone Fernández Valladares, ob. cit., n. ${ }^{\circ} 583$.

${ }^{59}$ Facsímil en Pliegos poéticos espanoles en la Universidad de Praga, intr. de Ramón Menéndez Pidal, Madrid, Joyas Bibliográficas, 1961, n. ${ }^{\circ} 22$.

${ }^{60}$ Lamarca, ob. cit., n. ${ }^{\circ} 215$.

${ }^{61}$ Facsímil en Los pliegos poéticos de la colección del Marqués de Morbecq, siglo XVI, ed. de Antonio Rodríguez-Moñino, Madrid, Estudios Bibliográficos, 1962, n. ${ }^{\circ} 5$.
} 
con RM1062 y RM1064 se ha supuesto que sería un pliego de dos hojas con igual contenido que estos y por ello catalogado como tal. Montserrat Lamarca, por su parte, lo ha considerado probable continuación de las pocas hojas conservadas del Libro de cincuenta romances, impreso por el propio Amorós en esos mismos años ${ }^{62}$. Como se sabe, de dicho Libro, que en realidad sería como mínimo una segunda edición, pues afirma en el título añadir una serie de textos, se conservan únicamente las cuatro primeras hojas, procedentes de la colección del Marqués de Morbecq, que en su día fue del Duque de T'Serclaes de Tilly, quien la adquirió, como lote completo, al librero muniqués Jaques Rosenthal. La hoja que nos ocupa, RM1063, también formaba parte de ese lote, en el que -de nuevo- abundan sobremanera los impresos bifolios y que, además, se caracteriza por un fuerte desorden, lo que no hace nada improbable que esta hoja pudiera ser, en efecto, parte del Libro. Ahora bien, lo que no se había notado hasta ahora, es que cuando en el vuelto de la primera hoja conservada del Libro se mencionan los añadidos, el romance de Guarinos y los de Gayferos aparecen nombrados uno detrás del otro:

Aqui comiençan cincuenta romāces el los cuales han (sic) añadidos los siguientes romāçes. El romance de Calisto y Melibea. Otro q comiença En las salas de Paris. Otro d Guarinos. Otro de Gayferos. Otro al conde de Oliua. Otro dl conde Claros. Otros tanbien de amores. Otro de la reyna helena. Otros de paris y las tres deeses. Y muchos otros romances ${ }^{63}$.

Según he argumentado en otro trabajo, todo parece indicar que Amorós utilizó para esas adiciones una serie de pliegos sueltos entre los que ya estaría -en torno a 1525- un pliego de cuatro hojas con esos dos (supuestos) bifolios $^{64}$. Este hecho, unido a la presencia contigua de ambos impresos en la colección de Campo de Alanje y el pliego tardío salido del taller de Felipe de Junta demuestran la existencia de una larga tradición impresa conjunta.

Este hecho, como ya he dicho, me parece de mucha importancia, porque afecta al motor mismo de la transmisión textual: ya no hablamos de colecciones y su posible formación, sino de la recepción de esos textos por sus

\footnotetext{
${ }^{62}$ Registrado consecuentemente bajo la misma entrada: Lamarca, ob. cit., n. ${ }^{\circ} 215$.

${ }^{63}$ Como puede verse, se menciona únicamente el romance de Gaiferos, pero creo que estamos ante una errata, provocada por la numerosa presencia en los términos colindantes de palabras terminadas en -os. De hecho, en la última línea se mencionan otros de paris, cuando el romance de Paris y las tres deesas es únicamente uno, el que comienza Por una linda espesura. La cursiva en la cita es mía.

${ }^{64}$ Defendí esa hipótesis en una comunicación presentada durante el VI Congreso internacional del Romancero celebrado los 25, 26 y 27 de noviembre de 2019 en la Universidad Complutense de Madrid y pendiente de publicación. Resumo aquí las conclusiones que expongo con el detalle necesario en ese trabajo.
} 
propios impresores. Y es que tampoco aquí nos las habemos con un hecho aislado, al contrario, aunque por razones de espacio me limito a mencionar solamente algunos ejemplos más que sustenten mi argumentación.

Valga lo que sucede con RM1065, un pliego impreso en Burgos, en el taller de Fadrique Biel de Basilea, c. 1515-151965, es decir, en una fase también muy temprana en la difusión de estos impresos. El pliego contiene romances, en concreto, En misa está el emperador y Mala vistes los franceses, el romance de Guarinos que acabamos de ver en pliegos anteriores. El pliego tiene una estructura algo extraña, ya que contiene dos grabados interiores, algo poco frecuente entre los pliegos poéticos de esta centuria. El primero de los grabados aparece al vuelto de la segunda hoja, a pesar de que el primer romance no termina allí, sino en el recto de la tercera. En esa tercera hoja encontramos además, al final de la segunda columna, el título del segundo romance, mientras que al vuelto de la tercera encontramos, encabezando la hoja, el segundo de los grabados interiores. Lo extraño de estos grabados es menos su existencia que su posición en el impreso. No creo necesario mencionar que muchos de estos pliegos sueltos presentan un grabado en la portada, casi siempre bajo el título; sí quiero recordar, en cambio, que también conocemos algunos pliegos que cierran con uno de estos tacos xilográficos ${ }^{66}$, pero muy pocos con grabados interiores ${ }^{67}$. En un ámbito como el del pliego, en el que la absoluta mayoría de los conocidos - con independencia ahora de formato o número de hojas- busca el máximo aprovechamiento del espacio tipográfico, resulta muy extraño encontrar ahí esos grabados, más aún cuando uno de ellos, el segundo, aparece en la posición que le correspondería a uno que abriera pliego y cuando, además, conocemos -según hemos visto- pliegos bifolios con ese romance de Guarinos. La explicación que, en mi opinión, resulta más factible para explicar esa disposición tipográfica es que Biel de Basilea se estaba valiendo de un impreso de cuatro hojas que, por razones que hoy ignoramos, no pudo o quiso reproducir de tal modo que los dos bifolios quedaran como tales en el pliego. Aunque no conozcamos testimonios bifolios con el primero de los romances, En misa está el emperador, este tiene la longitud adecuada ( 240 versos en este testimonio) para ser incluido en ese espacio: en una caja de 32 versos, como la que usa Junta en RM1065, este romance ocuparía tres caras enteras, dejando libre en una de ellas (el recto de la primera hoja, se

\footnotetext{
${ }^{65}$ Julián Martín Abad, Post-incunables ibéricos, Madrid, Ollero \& Ramos, 2001, n. ${ }^{\circ}$ 1371, Fernández Valladares, ob. cit., n. ${ }^{\circ} 104$.

${ }^{66}$ Por ejemplo RM484, impreso según Mercedes Fernández Valladares, ob. cit., n. ${ }^{\circ} 402$, en Burgos, por Juan de Junta, después de 1550. Hay edición facsímil en Pliegos Praga, ob. cit., I, n. ${ }^{\circ} 11$.

${ }^{67}$ Uno de esos pocos casos en Pliegos Praga, ob. cit., n. ${ }^{\circ} 49$.
} 
entiende) espacio suficiente para un grabado y un título. No creo que resulte necesario exponer las razones por las que este pliego se ha considerado y catalogado como un impreso de cuatro hojas, pero me parece evidente a la luz de lo expuesto hasta aquí que de modo directo o indirecto ese impreso se compuso en base a otro que contendría dos potenciales bifolios.

Una problemática semejante puede verse también en otro caso, que nos lleva directamente a las reflexiones finales. Entre los pliegos poéticos custodiados en la Biblioteca Nacional de Madrid ${ }^{68}$ se conserva un pliego de dos hojas titulado Siguese un perque sobre la passion de Christo, RM1083, que contiene Salid hijas de Sion y las coplas Entre todos los nacidos. En la Biblioteca de Ajuda, por su parte, se conserva otro pliego de igual título, pero esta vez de cuatro hojas, RM1082 ${ }^{69}$. Además de las dos composiciones recién mencionadas, el pliego lisboeta incluye un Romance sobre la passion de christo que ocupa las dos últimas hojas. El recto de la tercera hoja lleva este título en una tipografía mayor y nada, absolutamente nada, impide considerarlo como un bifolio. Sin embargo Rodríguez-Moñino lo recogió como pliego de cuatro hojas, García de Enterría lo editó en facsímil como pliego de cuatro hojas y sin mencionar en ningún momento alguno estas características del pliego ${ }^{70}$, el Nuevo Diccionario lo registra como pliego de cuatro hojas y el Suplemento se limita a introducir una nueva referencia bibliográfica.... ¿Debemos seguir considerándolo pliego de cuatro hojas o contiene dos productos editoriales distintos?

La pregunta es extensible a muchos otros impresos y afecta a un aspecto esencial de la transmisión del pliego suelto poético en el primer siglo de la imprenta. Ya mencioné más arriba que no voy a entrar aquí a discutir cómo deben catalogarse este tipo de impresos, pero sí planteo abiertamente mis dudas sobre los criterios con que determinar qué es y qué no es un bifolio, cuándo constituye y cuándo no constituye un producto editorial. Creo que partiendo de los ejemplos que hemos expuesto en este trabajo podemos ver claramente que en el largo proceso crítico que ha supuesto la catalogación de los pliegos sueltos poéticos quinientistas la condición bifolia se ha otorgado (y rechazado) sin fundamentos teóricos demasiado estables; antes bien, se ha procedido partiendo de una compresión del fenómeno que priorizaba los aspectos materiales (no necesariamente tipográficos). Sin embargo, que un

\footnotetext{
${ }^{68}$ Pliegos Madrid, ob. cit., IV, n. ${ }^{\circ} 160$.

${ }^{69}$ Pliegos poéticos españoles en bibliotecas de Portugal, ed. de María Cruz García de Enterría, Madrid, Joyas Bibliográficas, 1982, n. ${ }^{\circ} 1$.

${ }^{70}$ Pliegos Portugal, ob. cit., n. ${ }^{0} 1$. Quien tenga la suerte de poder disponer de la edición facsímil con los pliegos en rama dentro de la carpeta podrá constatar fácilmente que la posibilidad de contemplarlos como dos pliegos independientes es evidente.
} 
impreso tenga dos hojas no resulta criterio suficiente para determinar que se trataba de un bifolio, ni tan solo si la primera de esas dos hojas reúne todas las características de una portada; del mismo modo, tampoco cuatro hojas son signo inequívoco de que no estamos ante dos obras independientes. Si muchos impresos de cuatro hojas fueron cortados dando lugar a dos bifolios solamente en un periodo muy avanzado de su transmisión, cuando la codicia bibliófila había convertido a estas obras en piezas muy cotizadas, ¿podemos hablar con propiedad de esas hojas como bifolios? O si los propios impresores y editores comprendieron y utilizaron lo que hoy catalogamos como dos bifolios como un único pliego, ¿tiene sentido hablar de dos productos editoriales en un producto tipográfico? La cuestión es compleja. Obviamente, cuando un mismo producto tipográfico contiene dos impresos idénticos, habría de otorgarse a esos impresos la condición de bifolios, aún cuando esa no sea la constitución material en que se ha transmitido. Pero en aquellos otros casos en que el impreso de cuatro hojas contiene entre ellas partes a priori distintas, creo que cabría hablar, más que de bifolios, de impresos de cuatro hojas que contenían hojas susceptibles de ser consideradas como impresos independientes $-\mathrm{y}$ convertidas en tales mediante corte. Algunos impresores y editores comprendieron esos impresos como cuatro hojas, otros los dividieron en bifolios y algunos de estos, a su vez, fueron a juntarse con otros para constituir un nuevo impreso de cuatro hojas. Si mantenemos la rigidez en la visión de estos impresos creo que pasamos por alto un factor clave en la transmisión impresa del pliego poético. La condición bifolia a la que aludo en el título, entendida como la individualización material de una parte de un producto tipográfico mayor, es, para muchos impresos, solamente una potencialidad.

Recibido: $11 / 01 / 2020$

Aceptado: 6/03/2020 
$\cos 8$

\section{LA CONDICIÓN BIFOLIA: LOS IMPRESOS DE DOS HOJAS Y LA TRANSMISIÓN DEL PLIEGO SUELTO POÉTICO}

RESUMEN: La reconstrucción y análisis de algunos volúmenes facticios antiguos de pliegos sueltos poéticos revela que muchos de los hoy catalogados como bifolios se transmitieron hasta fechas relativamente recientes formando una unidad material de cuatro hojas con otro bifolio. Este hecho, además de cuestionar los modos de identificación y registro actuales, permite comprender la transmisión de ciertos impresos, pues así, unidos, es también como los impresores y editores quinientistas recibieron estos pliegos.

Palabras Clave: Pliego suelto. Bifolios. Ecdótica. Poesía de cancionero. Romancero.

THE BIFOLIA CONDITION: THE TWO-LEAF PRINTS

AND THE TRANSMISSION OF THE POETIC CHAP-BOOKS

ABSTRACT: The reconstruction and analysis of some old factitious volumes of poetic chap-books reveals that many of those today classified as bifolia were transmitted to relatively recent dates forming a four-leaf material unit with another bifolio. This fact questions the current identification and registration modes for this bifolia and allows us to understand the transmission of certain chap-books, since this was also the way in which they were received by printers and editors.

KeYwords: Chap-book. Bifolia. Ecdotics. Cancionero poetry. Romancero. 